\title{
Molecular Detection of Carbapenemase-Encoding Genes in Multidrug-Resistant Acinetobacter baumannii Clinical Isolates in South Africa
}

\author{
Yaw Adjei Anane, ${ }^{1}$ Teke Apalata $\mathbb{D}^{1,2}$ Sandeep Vasaikar ${ }^{1 D},{ }^{1,2}$ Grace Emily Okuthe, ${ }^{3}$ \\ and Sandile Songca ${ }^{4}$ \\ ${ }^{1}$ Division of Medical Microbiology, Department of Laboratory Medicine \& Pathology, Faculty of Health Sciences, \\ Walter Sisulu University, Private Bag: X1, Mthatha 5117, Eastern Cape Province, South Africa \\ ${ }^{2}$ Division of Medical Microbiology, National Health Laboratory Services (NHLS), Nelson Mandela Central Hospital, \\ Mthatha 5100, South Africa \\ ${ }^{3}$ Department of Biological \& Environmental Sciences, Walter Sisulu University, Private Bag: X1, Mthatha-5117, \\ Eastern Cape Province, South Africa \\ ${ }^{4}$ School of Chemistry and Physics, College of Agriculture Engineering and Science, University of KwaZulu-Natal, 2nd Floor, \\ Francis Stock Building, Howard College Campus, UKZN, Durban 4041, South Africa
}

Correspondence should be addressed to Sandeep Vasaikar; sandeepvasaikar@yahoo.com

Received 27 September 2019; Revised 17 February 2020; Accepted 13 March 2020; Published 13 June 2020

Academic Editor: Karl Drlica

Copyright (c) 2020 Yaw Adjei Anane et al. This is an open access article distributed under the Creative Commons Attribution License, which permits unrestricted use, distribution, and reproduction in any medium, provided the original work is properly cited.

Introduction. Carbapenem-resistant Acinetobacter baumannii has been responsible for an increasing number of hospital-acquired infections globally. The study investigated the prevalence of carbapenemase-encoding genes in clinical multidrug-resistant $A$. baumannii strains. Materials and Methods. A total of 100 nonduplicate multidrug-resistant A. baumannii strains were cultured from clinical samples obtained from healthcare facilities in the O. R. Tambo district. The strains were confirmed by detecting the intrinsic $b l a_{\text {OXA-51-like }}$ gene. Antimicrobial susceptibility testing was performed by VITEK ${ }^{\circledast} 2$ and autoSCAN-4 systems. The MIC of imipenem and meropenem was rechecked by E-test. Colistin MIC was confirmed by the broth microdilution method. Real-time PCR was performed to investigate the presence of carbapenemase-encoding genes. Results. Most strains showed high resistance rates $(>80 \%)$ to the antibiotics tested. Resistance to amikacin, tetracycline, and tigecycline were $50 \%, 64 \%$, and $48 \%$, respectively. All strains were fully susceptible to colistin. The $b l a_{\text {OXA-51-like }}$ was detected in all strains whilst $b l a_{\text {OXA-23-like, }}, b l a_{\text {OXA-58-like, }}, b l a_{\text {OXA- }}$ 24-like, $b l a_{\mathrm{IMP}-1}, b l a_{\mathrm{VIM}}$, and $b l a_{\mathrm{NDM}-1}$ were found in $70 \%, 8 \%, 5 \%, 4 \%, 3 \%$, and $2 \%$ of strains, respectively. None of the tested strains harboured the genes $b l a_{\mathrm{SIM}}$ and $b l a_{\mathrm{AmpC}}$. The coexistence of $b l a_{\mathrm{OXA}-23-\text { like, }}$ and $b l a_{\mathrm{IMP}-1}$ or $b l a_{\mathrm{OXA}-58-\mathrm{like}}$ was detected in $1 \%$ and $2 \%$ strains, respectively. A distinct feature of our findings was the coharbouring of the genes $b l a_{\mathrm{OXA}-23-\mathrm{like}}, b l a_{\mathrm{OXA}-58-\mathrm{like}}$, and $b l a_{\mathrm{IMP}-1}$ in $2 \%$ strains, and this is the first report in the Eastern Cape Province, South Africa. The intI1 was carried in $80 \%$ of tested strains whilst ISAba1/bla $a_{\text {OXA-51-like }}$ and ISAba1/bla $a_{\text {OXA-23-like }}$ were detected in $15 \%$ and $40 \%$ of the strains, respectively. The detection of $b l a_{\text {OXA-23-like, ISAba1/bla }}$ OXA-51-like, ISAba1/bla $a_{\text {OXA-23-like, }}$ and $b l a_{\text {OXA-23-like, }}, b l a_{\text {OXA-58-like, }}$ and $b l a_{\text {IMP-1 }}$ carbapenemases in strains had a significant effect on both imipenem and meropenem MICs. Conclusions. Results showed a high level of oxacillinases producing A. baumannii circulating in our study setting, highlighting the need for local molecular surveillance to inform appropriate management and prevention strategies. 


\section{Introduction}

Acinetobacter baumannii, an opportunistic Gram-negative bacterium, has become a predominant cause of healthcareassociated infections worldwide [1]. This microorganism has emerged to become a major concern for clinicians worldwide causing infections such as septicaemia, meningitis, endocarditis, ventilator associated pneumonia, burns, urinary tract infections, surgical site infections, and wound infections in hospitals due to its intrinsic as well as its remarkable propensity to rapidly acquire resistance determinants to a wide range of antibacterial agents $[2,3]$. The World Health Organization (WHO) lists A. baumannii among critical antibiotic-resistant "priority pathogens," highlighting its serious threats to public health [4]. Risk factors for A. baumannii infections include immune suppression, burns, trauma, mechanical ventilation, catheters, invasive medical procedures, previous antibiotic treatment, an extended hospital stay (>90 days), and underlying diseases such as diabetes [5].

Carbapenem has been the drug of choice used to treat MDR A. baumannii infections over the past decade because they are highly efficacious and have low toxicity [6]. However, the resistance rate against carbapenem has increased dramatically due to its frequent application in recent years and is mainly mediated by the production of carbapenem-hydrolysing enzymes [7]. The most common mechanism of carbapenem resistance in A. baumannii is the production of $\beta$-lactamases, including enzymes of Ambler Classes D OXA-type carbapenemases consisting of $b l a_{\text {OXA-23-like }}$ OXA-23, OXA-27 and OXA-49), bla $a_{\text {OXA-40- }}$ like, (OXA-24/40, OXA-25, OXA-26 and OXA-72), bla $a_{\text {OXA-58-like }}$ (OXA-58 and OXA-96), bla $a_{\text {OXA-143-like }}$ and $b l a_{\text {OXA-51-like }}$ enzymes, and B Metallo- $\beta$-lactamases (MBLs) mainly $b l a_{\mathrm{VIM}}, b l a_{\mathrm{IMP}-1}, b l a_{\mathrm{NDM}-1}, b l a_{\mathrm{SPM}}, b l a_{\mathrm{GIM}}$, and $b l a_{\mathrm{SIM}}$ types [8]. Among the multiple types of MBL genes described throughout the world, the $b l a_{\mathrm{IMP}-1}, b l a_{\mathrm{VIM}}$, and $b l a_{\mathrm{SIM}}$ types are the most common [9]. The upstream of OXA-type class $\mathrm{D}$ carbapenemases in Acinetobacter is often associated with insertion sequence (IS), of which ISAbal is the most commonly detected. ISAba1 and other IS may modulate the expression and transfer of OXA-type carbapenemase genes [10]. Integrons have been demonstrated to play a major role in the dissemination of multidrug-resistance among Gramnegative bacteria through the capture and expression of resistant genes embedded within cassettes [11].

Studies in Africa and the Gulf Cooperation Council (e.g., Saudi Arabia) have reported increasing carbapenem resistance among A. baumannii isolates [12-14]. Since nosocomial A. baumannii infection has generated an abundance of therapeutic obstacles for the treatment of hospitalized patients in South Africa, as well as other countries, the knowledge regarding the prevalence level of antibiotic resistance genes and the resistance pattern of this bacterium to the initial antibiotics is required to control, prevent, and cure A. baumannii driven infections. Therefore, the aim of the current study was to investigate the rates of carbapenem resistance as well as to detect the frequency of carbapenemase-encoding genes in MDR A. baumannii isolates obtained from hospitalized patients receiving treatment in healthcare facilities in O.R. Tambo district in the rural Eastern Cape Province of South Africa.

\section{Materials and Methods}

2.1. Study Design and Settings. This was a prospective crosssectional study conducted at healthcare facilities in the OR Tambo district municipality, Eastern Cape, South Africa. The municipality is formed by five local municipalities (King Sabata Dalindyebo, Nyandeni, Mhlontlo, Port St Johns, and Ingquza Hill) with an estimated total population of $1,760,389$. Healthcare services are delivered by 1 Academic Central Hospital, 1 Regional Hospital, 12 District Hospitals; 11 Community Health Centers, 49 clinics, 52 Health Posts, and 15 mobiles. Patients' clinical samples collected from all these facilities are sent for culture and susceptibility testing in the Department of Medical Microbiology at the National Health Laboratory Services (NHLS), located in the Nelson Mandela Central Hospital in Mthatha, Eastern Cape. Clinical samples from those various hospitals and clinics were sent as part of the patients' routine standard of care.

\subsection{Data Collection, Bacterial Identification, and Storage of A.} baumannii Isolates. From August 2016 to July 2017, a total of 100 nonduplicate nonconsecutive MDR A. baumannii strains were collected arbitrarily from blood, pus, urine, sputa, catheter tips, tracheal aspirates, and cerebrospinal fluid of patients from the Microbiology Laboratory at the National Health Laboratory Services (NHLS) at NMAH, Mthatha (see Supplementary Materials). The strains were preliminarily speciated phenotypically using standard microbiological procedures: the VITEK ${ }^{\circledR} 2$ automated machine with Gram-negative identification (GNI) cards (BioMérieux, France) and the MicroScan autoSCAN-4 System (Dade Behring Inc., Deerfield, IL) with Gram-negative ID type 2 panel. A high percentage $(\leq 95 \%)$ was utilized as the acceptance criterion for identification by the MicroScan autoSCAN system. Suspected colonies were also further verified using the Acinetobacter specific primer set Ac436F and Ac676r to amplify the $16 \mathrm{~S}$ rRNA gene. Confirmation of A. baumannii strains was carried out by polymerase chain reaction analysis of the presence of inherent $b l a_{\mathrm{OXA}-51-l i k e}$ genes. All of the strains were stored at $-80^{\circ} \mathrm{C}$ in skim milk with $15 \%$ glycerol until further use.

2.3. Antimicrobial Susceptibility Testing. A total of 18 clinically relevant antibiotics were tested using MicroScan autoSCAN-4 Gram-negative MIC panel, and results were analyzed and interpreted according to the recommended clinical breakpoints given in the clinical and laboratory standard institutes (CLSI) guidelines v27 [15]. These antibiotics were amikacin (AMK), ampicillin/sulbactam (AMS), cefepime (FEP), cefotaxime (CTX), ceftazidime (CAZ), ceftriaxone (CRO), ciprofloxacin (CIP), gentamicin (GEN), imipenem (IMP), meropenem (MEM), levofloxacin (LVX), tetracycline (TEC), tobramycin (TOB), trimethoprim/sulfamethoxazole (SXT), piperacillin/tazobactam (TZP), 
TABLE 1: Quality control (QC) organisms used for the detection of resistant genes in this study.

\begin{tabular}{|c|c|c|}
\hline Organism & ATCC or NCTC number & Resistant gene(s) \\
\hline A. baumannii & NCTC 13302 & OXA-26 (OXA-24-like; OXA-51-like \\
\hline A. baumannii & NCTC 13305 & OXA-58 \\
\hline Klebsiella pneumoniae & ATCC-BAA 2146 & NDM \\
\hline A. baumannii & NCTC 13301 & OXA-23 \\
\hline A. baumannii & NCTC 13303 & OXA-26 (OXA-51-like) \\
\hline A. baumannii & NCTC 13304 & OXA-27 (OXA-51-like) \\
\hline Enterobacter cloacae & NCTC 13405 & $\mathrm{AmpC}$ \\
\hline Pseudomonas aeruginosa & NSCC stock & VIM \\
\hline A. baumannii & ATCC 19606 & OXA-51 \\
\hline Escherichia coli & NCTC 13476 & IMP-1 \\
\hline Escherichia coli & Se 131 (accession number: AJ238350) & intI1 \\
\hline Pseudomonas aeruginosa & ATCC 27853 & OXA-24-like; OXA-51-like \\
\hline Klebsiella pneumoniae & ATCC 8303 & SIM-1 \\
\hline A. baumannii & AYE strain & AmpC, ISAba1, OXA-51-like \\
\hline
\end{tabular}

piperacillin (PRL), colistin (CST), and tigecycline (TGC), all from Beckman Coulter, South Africa. Determination of MICs to imipenem and meropenem rechecked by E-test minimum inhibitory concentration method using E-test strips as per the manufacturer's guidelines (BioMérieux, France) on Mueller-Hinton agar plates and CLSI breakpoints (strains displaying MICs $\geq 8 \mu \mathrm{g} / \mathrm{mL}$ for imipenem and meropenem were considered resistant). Quality control strains used in antimicrobial susceptibility testing are Escherichia coli ATCC \#25922, Staphylococcus aureus ATCC 29213, and Pseudomonas aeruginosa ATCC \#27853 [16]. Colistin susceptibility testing was confirmed using the broth microdilution method according to the CLSI and by ComASP $^{\mathrm{TM}}$ Colistin, Liofilchem ${ }^{\circledR}$ (REF. 75001), according to the manufacturer's recommendations. Escherichia coli NCTC 13846 ( $m c r-1$ positive) was used as a positive control for ComASP ${ }^{\mathrm{TM}}$ Colistin test. The United States Food and Drug Administration (US-FDA) approved breakpoints for members of the family Enterobacteriaceae were used as interpretative criteria for tigecycline (http://www.fda.org.uk/ sitemap.aspx). Nonsusceptibility was defined as a combination of resistance and intermediate resistance. MDR $A$. baumannii isolates were defined as acquired nonsusceptibility to at least one agent in three or more antimicrobial categories [17]. All results were within quality control ranges.

2.4. Detection of Carbapenem-Resistant Genes. Genomic DNA from an overnight culture on tryptic soy broth of $A$. baumannii was extracted using the MagNaPure Compact ${ }^{\otimes}$ nucleic acid isolation kit I (ref. no. 03730964001, Roche Diagnostics, Mannheim, Germany) according to the manufacturer's protocol. A final elution volume of $200 \mu \mathrm{L}$ of pure A. baumannii DNA was used. $2 \mu \mathrm{L}$ of the DNA extract was used for each PCR analysis. The extracted DNA was stored at $-80^{\circ} \mathrm{C}$ until required for further analysis. All MDR isolates were systematically screened by LightCycler 2.0 real-time PCR (Roche Diagnostics) instrument targeting some of the epidemiologically most relevant resistant genes. The presence of the genes encoding Amber class D Serine-Carbapenemase genes ( $b l a_{\text {OXA-51-like }}, b l a_{\text {OXA-23-like, }}, b l a_{\text {OXA-24-like, }}$

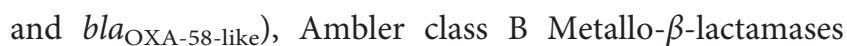
(MBLs) $\left(b l a_{\mathrm{IMP}-1}, b l a_{\mathrm{VIM}}, b l a_{\mathrm{SIM}}\right.$, and $\left.b l a_{\mathrm{NDM}-1}\right)$, and Ambler class $\mathrm{C} b l a_{\mathrm{AmpC}}$ were assessed. MDR A. baumannii strains were subsequently screened for the presence or absence of IS element ISAba1 [18] and class 1 integrons by real-time PCR [19]. To determine the overexpression of bla $a_{\text {OXA-23-like }}$ and

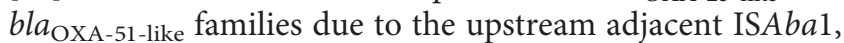
PCR was firstly performed for ISAba1, and in cases where $b l a_{\text {OXA-like }}$ is present, a combination of forward primer of ISAbal and reverse primer of $b l a_{\text {OXA-like }}$ family was further used. Appropriate positive and negative controls were run simultaneously (Table 1). The primers used for PCR amplification of the carbapenemase genes are listed in Table 2.

2.5. Statistical Analyses. Data analyses were performed using the Statistical Package for Social Sciences Statistics for Windows software package version 23.0 (IBM, Armonk, NY). Results were expressed using frequency and percentages for qualitative variables and as mean (standard deviation) or median (interquartile range: IQR) for quantitative variables. The association between A. baumannii and drug resistance was analyzed by the chi-square test. All $P$ values were two-sided. A $P$-value $<0.05$ was considered statistically significant.

2.6. Ethical Considerations. Ethical approval for this project was obtained from the Walter Sisulu University Research Ethics and Biosafety Committee (Reference number: 019/ 2016). No written informed consent was required because each MDR A. baumannii isolate obtained was delinked with the patient's personal information. Also authors had no direct interactions with patients from whom these isolates were isolated.

\section{Results}

3.1. Demographic Characteristics of the Study Population. The demographic characteristics of the patients with $A$. baumannii infection analyzed in our study showed a slight female preponderance of $47 \%$ males versus 53\% females). The age of the overall patients ranged between 1 day and 91 
TABLE 2: List of primers used for PCR amplification of resistant genes.

\begin{tabular}{|c|c|c|c|c|}
\hline Gene & Oligonucleotide sequence & Fragment size (bp) & Location & References \\
\hline 16SrRNA & $\begin{array}{l}\text { F: 5'TTT AAG CGA GGA GGA GG3' } \\
\text { R: 5'ATT CTA CCA TCC TCT CCC3' }\end{array}$ & 240 & $16 S r R N A$ & {$[20]$} \\
\hline OXA-23-like & $\begin{array}{l}\text { F: } 5^{\prime} \text { GATCGGATTGGAGAACCAGA3' } \\
\text { R: 5'ATTTCTGACCGCATTTCCAT3' }\end{array}$ & 501 & $b l a_{\mathrm{OXA}-23}$ & {$[21]$} \\
\hline OXA-24-like & $\begin{array}{l}\text { F: 5'GGTTAGTTG GCC CCC TTA AA3' } \\
\text { R: 5'AGTTGAGCGAAAAGGGGATT3' }\end{array}$ & 246 & $b l a_{\mathrm{OXA}-24}$ & {$[21]$} \\
\hline OXA-51-like & $\begin{array}{l}\text { F: 5'TAATGCTTT GATCGG CCT TG3' } \\
\text { R: 5'TGGATTGCACTT CAT CTT GG3' }\end{array}$ & 353 & $b l a_{\mathrm{OXA}-51}$ & {$[21]$} \\
\hline OXA-58-like & $\begin{array}{l}\text { F: 5'AAGTATTGGGGCTTGTGCTG-3' } \\
\text { R: 5'CCCCTCTGCGCTCTACATAC3' }\end{array}$ & 599 & $b l a_{\mathrm{OXA}-58}$ & {$[21]$} \\
\hline IMP-1 & $\begin{array}{l}\text { F: 5'GATGGTATGGTGGCTCTTGT3' } \\
\text { R: 5'TTAATTTGCCGGACTTAGGC3' }\end{array}$ & 448 & $B l a_{\mathrm{IMP}}$ & {$[22]$} \\
\hline NDM-1 & $\begin{array}{l}\text { F: 5'ATTAGCCGCTGCATTGAT3' } \\
\text { R: 5'CATGTCGAGATAGGAAGTG3' }\end{array}$ & 154 & $b l a_{\mathrm{NDM}}$ & {$[23]$} \\
\hline VIM-like & $\begin{array}{l}\text { F: 5'ACTCACCCCCATGGAGTTTT3' } \\
\text { R: 5'ACGACTGAGCGATTTGTGTG3' }\end{array}$ & 815 & $b l a_{\mathrm{VIM}}$ & {$[24]$} \\
\hline SIM-1-like & $\begin{array}{l}\text { F: 5'TAATGCTTT GATCGG CCT TG3' } \\
\text { R: 5'TGGATTGCACTT CAT CTT GG3' }\end{array}$ & 353 & $b l a_{\mathrm{SIM}}$ & {$[21]$} \\
\hline AmpC & $\begin{array}{l}\text { F: ACAGAGGAGCTAATCATGCG } \\
\text { R: GTTCTTTTAAACCATATACC }\end{array}$ & 1243 & $b l a_{\mathrm{AmpC}}$ & {$[25]$} \\
\hline$I S A b a 1$ & $\begin{array}{l}\text { F: 5-CACGAATGCAGAAGTTG-3 } \\
\text { R: 5-CGACGAATACTATGACAC-3 }\end{array}$ & 599 & ISAba 1 & {$[18]$} \\
\hline intI1 & $\begin{array}{l}\text { F: } 5^{\prime} \text { CAG TGG ACA TAA GCC TGT TC3 } \\
\text { R: } 5^{\prime} \text { CCC GAC GCA TAG ACT GTA3 }\end{array}$ & 160 & Integrase gene & {$[19]$} \\
\hline
\end{tabular}

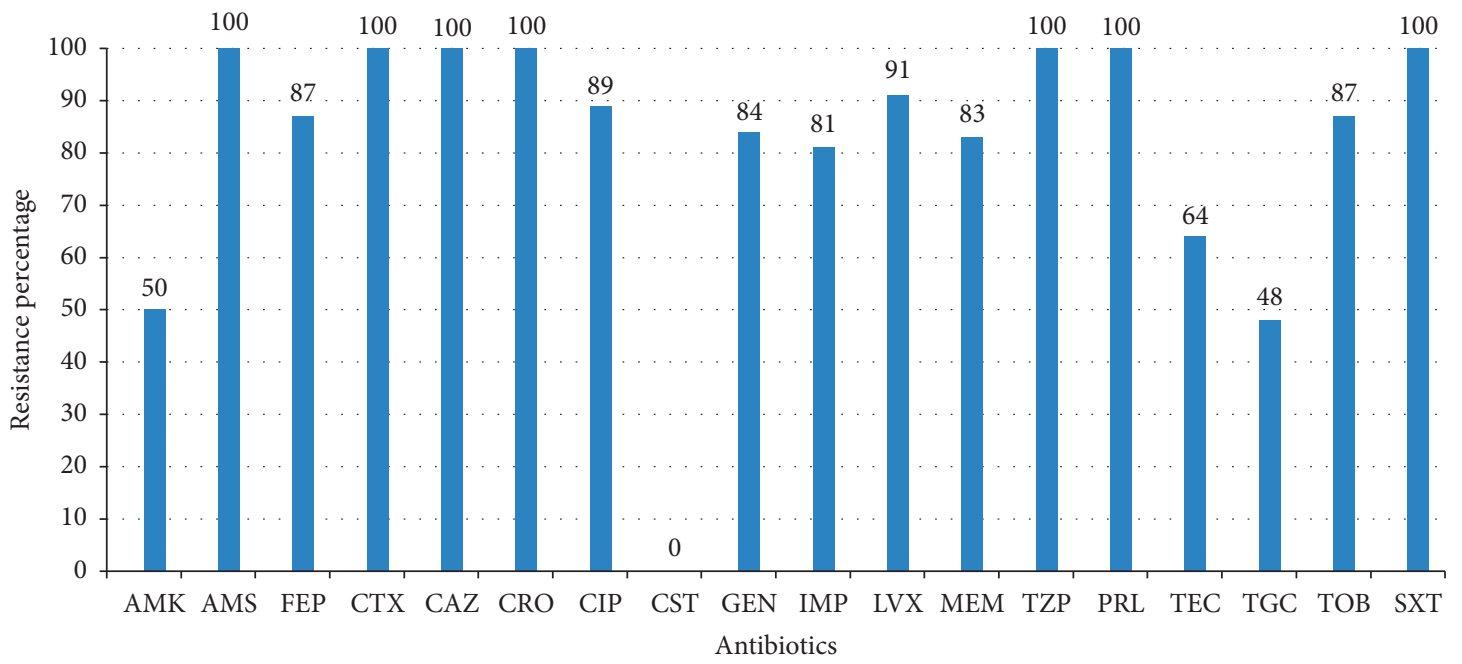

FIGURE 1: Antimicrobial resistance profile of $100 \mathrm{~A}$. baumannii isolates. IMP: imipenem; MEM: meropenem; FEP: cefepime; CAZ: ceftazidime; CTX: cefotaxime; CRO: ceftriaxone; AMS: ampicillin/sulbactam; PRL: piperacillin; TZP: piperacillin/tazobactam; AMK: amikacin; TOB: tobramycin; GEN: gentamicin; TEC: tetracycline; CIP: ciprofloxacin; LVX: levofloxacin; SXT: trimethoprim-sulfamethoxazole; TGC: tigecycline; CST: colistin.

years (mean 44.4 years, $S D \pm 36.3$ years). A. baumannii were isolated highest from tracheal aspirate (32\%) and lowest from cerebrospinal fluid (2\%). A. baumannii was most prevalent in the ICU wards $41 \%$ followed by neonatal ward $22 \%$, surgical ward $15 \%$, medical ward $13 \%$, and general ward $9 \%$. A. baumannii was isolated from various age groups but was the most prevalent in age group 0-9 years (Supplementary Materials).
3.2. Antimicrobial Susceptibility Testing. Generally, the results for antimicrobial susceptibility testing for the $100 \mathrm{MDR}$ A. baumannii revealed that $A$. baumannii strains showed a high resistance rate to most of the commonly used antibiotics tested. Overall, $\geq 81 \%$ of all the strains were resistant to extended-spectrum cephalosporins, fluoroquinolones, and carbapenems antibiotics. Resistance to aminoglycosides ranges from $50 \%$ to $87 \%$ whilst $48 \%$ of strains were resistant 


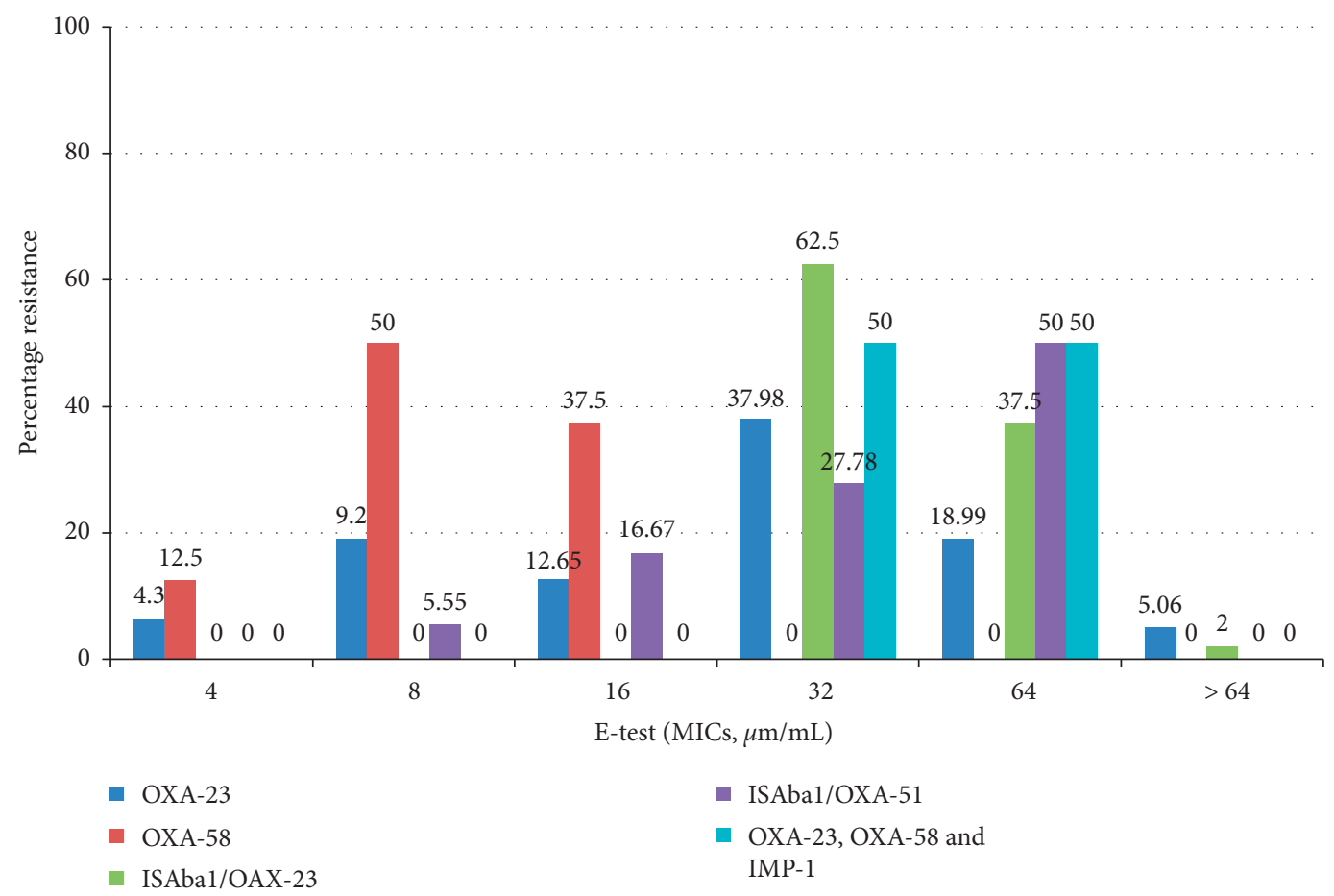

FIgURE 2: Distribution of imipenem MICs in $100 \mathrm{CRAB}$ isolates with the $b l a_{\text {OXA-23-like, }}, b l a_{\text {OXA-58-like, ISAba1/bla }}$ OXA-23-like, ISAba1/ $b l a_{\mathrm{OXA}-51-l i k e}$ and $b l a_{\mathrm{OXA}-23-\mathrm{like}}, b l a_{\mathrm{OXA}-58-\text { like }}$ and $b \operatorname{la}_{\mathrm{IMP}-1}$ genes.

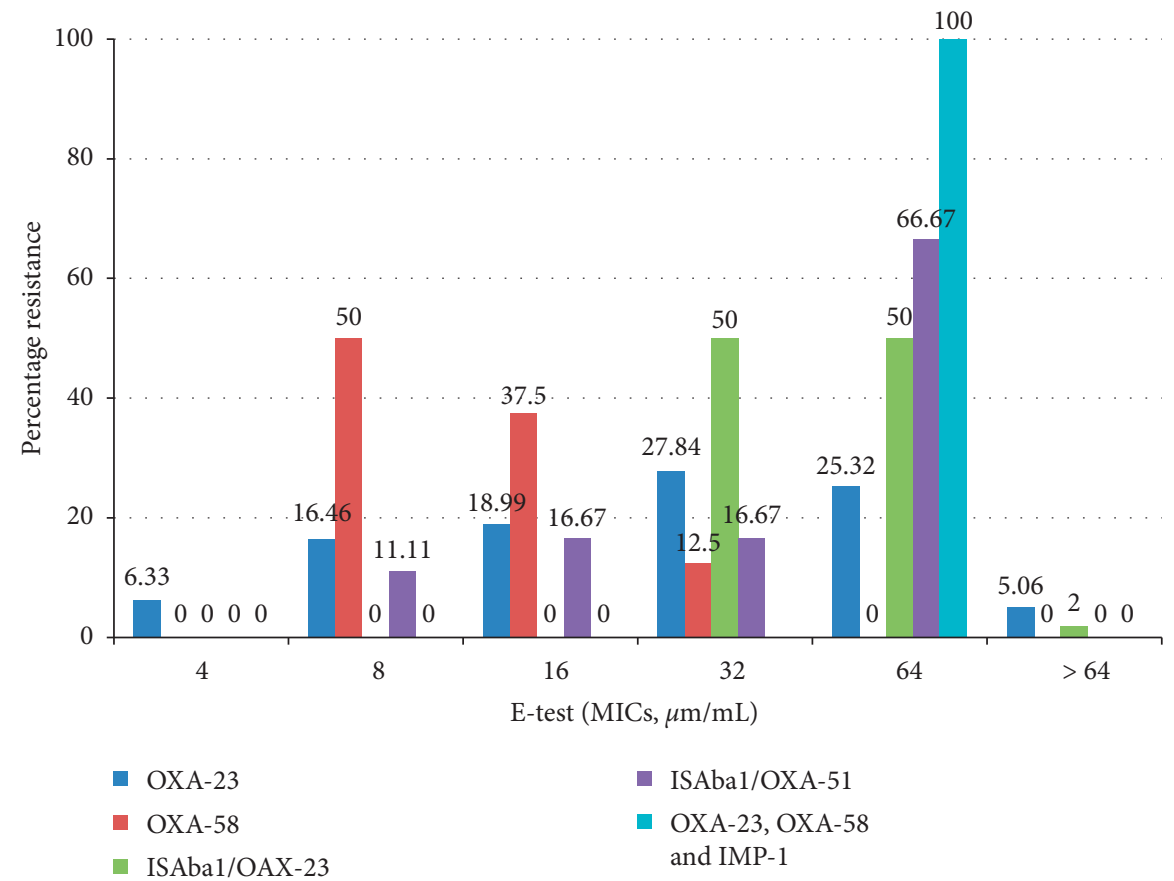

FIGURE 3: Distribution of meropenem MICs in 100 CRAB isolates with the $b l a_{\text {OXA-23-like, }} b l a_{\text {OXA-58-like, ISAba1/blaxA-23-like, ISAba1/ }}$ $b l a_{\text {OXA-51-like }}$ and $b l a_{\text {OXA-23-like, }} b l a_{\text {OXA-58-like }}$ and $b l a_{\text {IMP-1 }}$ genes.

to tigecycline. All strains were susceptible to colistin. Findings of the antimicrobial susceptibility patterns of all $A$. baumannii strains in this study are shown in Figure 1.
3.3. PCR Results of Carbapenemase-Encoding Genes. Results showed that all of the strains investigated in this study had the intrinsic $b l a_{\text {OXA-51-like }}$ gene. Among them, $70 \%$ were positive for the $b l a_{\mathrm{OXA}-23-\text { like }}, 8 \%$ were positive for 
TABLE 3: Percentage distribution of carbapenemases-encoding genes in 100 CRAB strains.

\begin{tabular}{|c|c|}
\hline Carbapenemases-encoding genes & Number (\%) \\
\hline$b l a_{\text {OXA-51-like }}$ only & 0 \\
\hline$b l a_{\text {OXA-51-like }}$ and $b l a_{\text {OXA-23-like }}$ & 23 \\
\hline$b l a_{\text {OXA-51-like }}$ and $b l a_{\text {OXA-58-like }}$ & 4 \\
\hline$b l a_{\text {OXA-51-like }}$ and $b l a_{\text {OXA-24-like }}$ & 5 \\
\hline$b l a_{\mathrm{OXA}-51-\text { like }}$ and $b l a_{\mathrm{IMP}-1}$ & 1 \\
\hline$b l a_{\mathrm{OXA}-51-\text { like }}$ and $b l a_{\mathrm{VIM}}$ & 3 \\
\hline$b l a_{\mathrm{OXA}-51-\text { like }}$ and $b l a_{\mathrm{NDM}-1}$ & 2 \\
\hline ISAba1/bla OXA-51-like & 15 \\
\hline$b l a_{\mathrm{OXA}-51-\text { like }}$ and ISAba1/bla $a_{\mathrm{OXA} 23 \text {-like }}$ & 40 \\
\hline$b l a_{\text {OXA-51-like }}$ and $b l a_{\text {OXA23-like }}$ and $b l a_{\mathrm{IMP}-1}$ & 1 \\
\hline$b l a_{\text {OXA-51-like }}$ and $b l a_{\text {OXA-23-like }}$ and $b l a_{\text {OXA-58-like }}$ & 2 \\
\hline$b l a_{\mathrm{OXA}-51-\text { like }}$ and $b l a_{\mathrm{OXA}-23-\text { like }}$ and $b l a_{\mathrm{OXA}-58 \text {-like }}$ and $b l a_{\mathrm{IMP}-1}$ & 2 \\
\hline ISAba1/bla $a_{\text {OXA-51-like }}$ and ISAba1/bla $a_{\text {OXA-23-like }}$ & 2 \\
\hline intI1 only & 0 \\
\hline
\end{tabular}

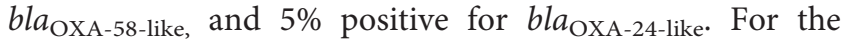
MBLs, $4 \%$ strains were positive for $b l a_{\mathrm{IMP}-1}, 3 \%$ carried $b l a_{\mathrm{VIM}}, 2 \%$ carried $b l a_{\mathrm{NDM}-1}$, and none of the tested isolates harboured the bla $a_{\mathrm{SIM}}$. Alleles encoding bla $a_{\mathrm{AmpC}}$ enzymes were not detected in any strain. The ISAbal was located upstream the $b l a_{\text {OXA-51-like and the bla }}$ OXA-23-like carrying strains $15 \%$ and $40 \%$, respectively. Some of the strains coharboured different carbapenemase genes mostly yielding very broad-spectrum antibiotic resistance profiles to imipenem and meropenem. Both strains possessing the ISAba1/ bla $a_{\mathrm{OXA}-23-\text { like }}$ and $b l a_{\mathrm{OXA}-23-\text { like, }}, b l a_{\mathrm{OXA}-58-\text { like, }}$ and $b l a_{\mathrm{IMP}-1}$ genes had the highest carbapenem resistance with these strains having imipenem MICs of $32 \mu \mathrm{g} / \mathrm{mL}$ or more, and having meropenem MICs of $32 \mu \mathrm{g} / \mathrm{mL}$ or more. This was

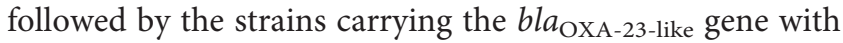
$62 \%$ having imipenem MICs of $32 \mu \mathrm{g} / \mathrm{mL}$ or more, and $58 \%$ having meropenem MICs of $32 \mu \mathrm{g} / \mathrm{mL}$ or more (Figures 2 and 3). Screening for the presence of integron integrase genes showed the presence of the class 1 integrase gene in 80\% MDR A. baumannii strains (Table 3).

\section{Discussion}

Understanding the fundamental mechanism that underlines A. baumannii infections, including original source of infecting strains, resistance patterns, and genes responsible for the development of resistance is critical for the development of appropriate infection control measures and more efficient treatment strategies [5]. Data from countries that lack routine surveillance, including South Africa, are necessary in order to understand and prevent the dissemination of carbapenem-resistant strains of species that are in epidemiological expansion, like A. baumannii.

Results from the present study revealed a $100 \%$ resistant rate of MDR A. baumannii to third-generation cephalosporins (ceftazidime, cefotaxime, and ceftriaxone). Resistance to cefepime was $87 \%$. All strains were resistant to piperacillin, piperacillin/tazobactam, trimethoprim/sulfamethoxazole, and ampicillin/sulbactam. The percentage rates of resistance to remaining antibiotics range from $48 \%$ to $89 \%$ with the exception of colistin which had no resistant strain detected.
Carbapenem resistance of $A$. baumannii is a major concern since it is the drug of choice in the treatment of $A$. baumannii infections [26]. The results showed that the majority of $A$. baumannii strains tested were resistant to both imipenem $81 \%$ and meropenem $83 \%$. Lowings et al. found all MDR $A$. baumannii isolates showed high level resistance (cefotaxime $100 \%$; cefotaxime $100 \%$; ceftazidime $89 \%$; cefepime $90 \%$; imipenem $86 \%$; and meropenem $86 \%$ ) in a study conducted at the Department of Medical Microbiology, Prinshof Campus, University of Pretoria/National Health Laboratory Service (NHLS) in 2013 [27]. A previous study by Kock et al. in the same setting showed that the overall percentage of resistance to the cephalosporin antibiotics was cefepime (62\%) and ceftazidime (45\%) [28]. A report on antimicrobial resistance surveillance from sentinel public hospitals in South Africa in 2013 showed a resistance rate of $80 \%$ to cefepime and $73 \%$ to ceftazidime [29]. Comparing the results of the present study with these earlier studies indicates that the resistant rate of MDR A. baumannii to various antibiotics has not decreased. High levels of resistance to third and fourth generation cephalosporins in this study indicated that cephalosporins are no longer efficacious in the treatment of MDR A. baumannii isolated in South Africa. The full susceptibility of all MDR $A$. baumannii to colistin in this study indicated that colistin is still a better option of the drug for the treatment of infections caused by A. baumannii in South African hospitals. However, the issue of nephrotoxicity, neurotoxicity, colistin-resistance, and heteroresistance shown by colistin monotherapy is a challenge in the management of this infection [30]. The high resistance rate to other antibiotic categories may probably be due to the heavy selection pressure from overuse of these antibiotics.

Although A. baumannii infections are observed in all body parts, they are mostly observed in the respiratory system. Our study showed that A. baumannii isolates were predominantly found in the respiratory samples (tracheal aspirate) and the most frequent setting for MDR A. baumannii was found in the ICUs (41\%). ICUs (Neonatal and general intensive care) are settings where most patients have indwelling devices and invasive procedures. Guckan et al. and Lowings et al. isolated $85.2 \%$ and $12 \% \mathrm{~A}$. baumannii isolates, respectively, from ICUs $[27,31]$. 
Molecular detection of carbapenemase-encoding genes has shown that the production of OXA-type carbapenemases is predominant in A. baumannii [30, 32]. The chromosomally encoded $b l a_{\text {OxA-51-like }}$ gene was detected in all the strains supporting those of other studies demon-

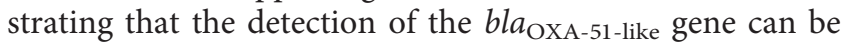
used as a supplementary tool to identify A. baumannii at the species level together with additional methods [33]. It was observed that the expression of $b l a_{\text {OXA-23-like was the second }}$ dominant carbapenem resistance mechanism (70\%) in MDR A. baumannii among the strains investigated aside the intrinsic $b l a_{\text {OXA-51-like gene. Its prevalence among isolates was }}$ higher than this in previous reports in South Africa. Its prevalence among strains was higher in this study than in the previous report (59\%) by Kock et al., whilst Lowings et al. reported a prevalence of $77 \%$. The $b l a_{\text {OXA-23-like gene is }}$ considered a virulence biomarker and a significant cause of carbapenem resistance worldwide [34]. This gene was first reported in 1985 in Scotland and since then outbreaks of OXA-23 carbapenemase-producing A. baumannii have been reported all over the world and is a dominant genetic determinant in the Asia subcontinent [35]. In Latin America, Brazil was the first country to report OXA-23-like-producing isolates in 2003, followed by Colombia, Argentina, and Mexico some years later [36].

This study also detected $b l a_{\text {OXA-24-like }}$ and $b l a_{\text {OXA-58-like }}$ genes in 5\% and $8 \%$ isolates, respectively. Although the acquired $b l a_{\text {OXA-23-like }}$ is the dominant genetic determinant reported in most studies in South Africa, the detection of bla $a_{\text {OxA- }}$

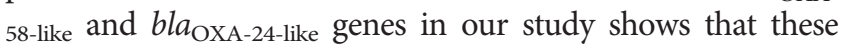
genes are becoming significant in carbapenem resistance in $A$. baumannii and may be responsible for the increase in carbapenem resistance in A. baumannii. It also suggested that these oxacillinases may locally spread in Mthatha, South Africa. These findings concur with a study by Ji et al. in China, who reported that $b l a_{\text {OXA-23-like }}$ are the most frequent carbapenemresistant $A$. baumannii, whereas $b l a_{\text {OXA-24-like }}$ and $b l a_{\text {OXA-58-like }}$ genes pose potential threats of hospital outbreaks of multidrugresistant $A$. baumannii [37]. For class B metallo- $\beta$-lactamases, we found that $4 \%, 3 \%$, and $2 \%$ of the isolates had $b l a_{\mathrm{IMP}-1}$, $b l a_{\mathrm{VIM}}$, and $b l a_{\mathrm{NDM}-1}$, respectively, and no isolate was found to produce $b l a_{\text {SIM }}$ gene. None of the clinical A. baumannii isolates harboured $b l a_{\text {AmpC }}$. IMP (primarily detected in South Korea) and NDM-1 (recently reported in many countries, such as India, Israel, Egypt, Germany, Spain, Switzerland, the United Arab Emirates, and China) are the two MBL genes most frequently detected in A. baumannii isolates [7, 33]. The bla $a_{\mathrm{NDM}-1}$ producing bacteria are mainly reported in Enterobacteriaceae [38]. This gene was first identified from Klebsiella pneumoniae and E. coli strains from a patient previously hospitalized in India and has recently emerged in A. baumannii isolates [32]. Taking into consideration the diversity of the South African community (prominence of Asian and European communities where $b l a_{\mathrm{NDM}-\mathrm{I}}$ have been reported) and the frequency of travels made by individuals between these countries, it is believed that the spread of $b l a_{\mathrm{NDM}-1}$ was likely to occur mostly through $A$. baumannii rather than Enterobacteriaceae. However, these findings need further investigation.
Interestingly, this study showed that some isolates were found to coharbour carbapenemase genes. Among these isolates include the coharbouring of carbapenemase genes $b l a_{\text {OXA- }}$ 23-like and $b l a_{\mathrm{IMP}-1}$ in $1 \%$ isolate, $b l a_{\mathrm{OXA}-23-\text { like }}$ and $b l a_{\mathrm{OXA}-58-\text { like }}$ in $2 \%$ isolates, and $b l a_{\mathrm{OXA}-23-\mathrm{like}}, b l a_{\mathrm{OXA}-58-\mathrm{like}}$, and $b l a_{\mathrm{IMP}-1}$ in $2 \%$ isolates. To the best of our knowledge, this is the first report of the coharbouring of $b l a_{\text {OXA-23-like, }} b l a_{\text {OXA-58-like, and } b l a_{\mathrm{IMP}-1}}$ in the Eastern Cape province of South Africa. We believe that coharbouring of these resistance genes is due to the presence of different genes in the same strain. This warrants the use of molecular typing methods to determine the clonal types of the isolates. The coharbouring of these major resistance mechanisms seriously limits therapeutic options, raising concerns regarding their transmission to other organisms. For example, we found that all isolates that coharboured $b l a_{\text {OXA-23-like, }}$

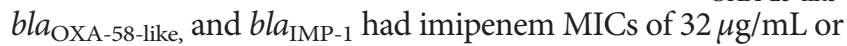
more and meropenem MICs of $64 \mu \mathrm{g} / \mathrm{mL}$.

The study showed that carbapenem susceptibility phenotypes of our A. baumannii isolates clearly correlated with the presence or absence, in the genomes, of ISAbal-linked $b l a_{\text {OXA- }}$

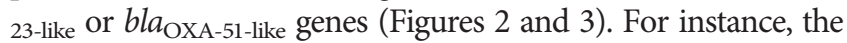
$100 \%$ MDR isolates with ISAba1 elements upstream of the bla $a_{\text {OXA-23-like }}$ had imipenem and meropenem MICs $32 \mu \mathrm{g} / \mathrm{mL}$ or more, whereas 83.34 and $77.78 \%$ of MDR isolates with ISAba1 elements upstream of the $b l a_{\text {OXA-51-like }}$ had imipenem and meropenem MICs $32 \mu \mathrm{g} / \mathrm{mL}$, respectively. Presumably, the ISAba1 element likely provides exogenous promoter functions to overexpress the linked genes. Evidence showed that carbapenem resistance mediated by overexpression of bla $a_{\text {OXA-like }}$ families due to upstream IS elements is more prevalent [39-42]. Our data are consistent with the findings of previous studies in that acquisition ISA $b a 1$ are the main mechanism of carbapenem resistance among $A$. baumannii by acting as a promoter to increase oxacillinase expression $[9,39,43]$. However, there are limited studies in South Africa as most of the studies evaluated the prevalence of OXA-like families in detail. There is, therefore, the need for further investigations to find the contribution of other IS elements (ISAba2, ISAba3) upstream to OXA-like families in different parts of the country. The results suggested that class 1 integrons did not contribute resistance to carbapenems since its presence did not have an effect on the MICs of carbapenems.

4.1. Limitations. The study has some limitations. Firstly, the study was conducted in a single centre, NMAH in the Tambo district municipality, so our findings may not be generalized to other settings. Secondly, the study did not include molecular typing epidemiological study reference methods such as PFGE, MLST, REP-PCR, etc. which would have better evaluated the dissemination of a carbapenemase or some clones to understand CRAB isolates in the clinical setting. These studies are necessary to help highlight the need for area-specific surveillance to inform appropriate management.

\section{Conclusion}

In conclusion, the results of this study provide direct evidence of the spread of carbapenemase-encoding genes 
especially $b l a_{\mathrm{OXA}-23 \text {-like }}$ in our setting. High presence of the ISAba 1 element upstream of the $b l a_{\text {OXA-51-like }}$ and $b l a_{\text {OXA-23- }}$ like increases expression of these carbapenemases and presents an emerging threat in this study setting. The results also provided clinically relevant insights into antimicrobial susceptibility and knowledge of the prevalent resistance mechanism among CRAB. This information will help in preventing the development of MDR A. baumannii in hospitals and also helps clinicians in the empirical treatment of patients. To prevent the development of Carbapenemresistant A. baumannii, routine implementation of simple and cost-effective screening methods to detect carbapenemase production in hospitals is crucial in enhancing infection control practices and finally establishing antimicrobial stewardship programs. Contribution and interplay of different mechanisms to the rapid increase in antibiotic resistance of A. baumannii in South Africa warrant further investigation.

\section{Data Availability}

All data generated or analyzed during this study are included in this published article (and its supplementary information file).

\section{Ethical Approval}

Ethical approval for this project was obtained from the Walter Sisulu University Research Ethics Committee (Human) (reference number: 019/2016).

\section{Conflicts of Interest}

The authors declare that there are no conflicts of interest regarding the publication of this paper.

\section{Authors' Contributions}

Songca S. P., Apalata T., Vasaikar S. D., and Anane Y. A. conceived and designed the experiments. Vasaikar S. D. and Anane Y. A. performed the experiments. Songca S. P., Apalata T., Vasaikar S. D., Anane Y. A., and Okuthe G. E. analysed the data. Songca S. P, Okuthe G. E., and Vasaikar S. D contributed reagents/materials/analysis tools. Songca S. P., Apalata T., Vasaikar S. D., and Anane Y. A. analysed data and wrote the manuscript. All authors read and approved the final manuscript.

\section{Acknowledgments}

The authors warmly acknowledge the host, Walter Sisulu University and the cohost, University of Zululand. Funding of the research through the National Research Foundation (NRF) Thuthuka (grant UID: 107619) is graciously appreciated. Beyond this, it did not take part in the research activities of knowledge creation and dissemination. The Nelson Mandela Academic Hospital (NMAH) and the Microbiology Laboratory at the National Health Laboratory Services (NHLS) at the NMAH are also acknowledged.

\section{Supplementary Materials}

Details of the genotypic features of Acinetobacter baumannii isolates consisting of genes encoding Amber class D serinecarbapenemase genes (blaOXA-51-like, blaOXA-23-like, blaOXA-24-like, and blaOXA-58-like), Amber class B metallo- $\beta$-lactamases (blaIMP-1, blaVIM, blaSIM, and blaNDM-1) and Amber class C blaAmpC. Age range, gender, ward, and isolate source are also indicated. (Supplementary Materials)

\section{References}

[1] A. Y. Peleg, H. Seifert, and D. L. Paterson, "Acinetobacter baumannii: emergence of a successful pathogen," Clinical Microbiology Reviews, vol. 21, no. 3, pp. 538-582, 2008.

[2] G. Huang, X. Shen, Y. Gong et al., "Antibacterial properties of acinetobacter baumanniiphage Abp1 endolysin (PlyAB1)," BMC Infectious Diseases, vol. 14, no. 1, p. 681, 2014.

[3] W. A. Warner, S. N. Kuang, R. Hernandez et al., "Molecular characterization and antimicrobial susceptibility of Acinetobacter baumannii isolates obtained from two hospital outbreaks in Los Angeles County, California, USA," BMC Infectious Diseases, vol. 16, no. 1, p. 194, 2016.

[4] Organization WHO, WHO Publishes List of Bacteria for Which New Antibiotics Are Urgently Needed, WHO, Geneva, Switzerland, 2017, http://www.who.int/mediacentre/news/ releases/2017/bacteria-antibiotics-needed/en/.

[5] S. El-Shazly, A. Dashti, L. Vali, M. Bolaris, and A. S. Ibrahim, "Molecular epidemiology and characterization of multiple drug-resistant (MDR) clinical isolates of Acinetobacter baumannii," International Journal of Infectious Diseases, vol. 41, pp. 42-49, 2015.

[6] N. Z. Ning, X. Liu, C. M. Bao et al., "Molecular epidemiology of blaOXA-23-producing carbapenem resistant Acinetobacter baumannii in a single institution over a 65 -month period in north China," BMC Infectious Diseases, vol. 17, no. 1, 2017.

[7] N. Karah, C. K. Dwibedi, K. Sjöström et al., "Novel aminoglycoside resistance transposons and transposon-derived circular forms detected in carbapenem-resistant Acinetobacter baumannii clinical isolates," Antimicrobial Agents and Chemotherapy, vol. 60, no. 3, pp. 1801-1818, 2016.

[8] D. M. Ghaith, M. M. Zafer, M. H. Al-Agamy, E. J. Alyamani, R. Y. Booq, and O. Almoazzamy, "The emergence of a novel sequence type of MDR Acinetobacter baumannii from the intensive care unit of an Egyptian tertiary care hospital," Annals of Clinical Microbiology and Antimicrobials, vol. 16, no. 1, p. 34, 2017.

[9] T.-H. Wang, Y.-S. Leu, N.-Y. Wang, C.-P. Liu, and T.-R. Yan, "Prevalence of different carbapenemase genes among carbapenem-resistant Acinetobacter baumannii blood isolates in Taiwan," Antimicrobial Resistance \& Infection Control, vol. 7, no. 1, p. 123, 2018.

[10] J. F. Turton, M. E. Ward, N. Woodford et al., "The role of ISAba1 in expression of OXA carbapenemase genes in Acinetobacter baumannii," FEMS Microbiology Letters, vol. 258, no. 1, pp. 72-77, 2006.

[11] E. Couvé-Deacon, T. Jové, P. Afouda et al., "Class 1 integrons in Acinetobacter baumannii: a weak expression of gene cassettes to counterbalance the lack of LexA-driven integrase repression," International Journal of Antimicrobial Agents, vol. 53, no. 4, pp. 491-499, 2019. 
[12] L. Al-Hassan, H. El Mehallawy, and S. G. B. Amyes, "Diversity in Acinetobacter baumannii isolates from paediatric cancer patients in Egypt," Clinical Microbiology and Infection, vol. 19, no. 11, pp. 1082-1088, 2013.

[13] A. A. Alsultan, B. A. Evans, E. A. Elsayed et al., "High frequency of carbapenem-resistant Acinetobacter baumannii in patients with diabetes mellitus in Saudi Arabia," Journal of Medical Microbiology, vol. 62, no. 6, pp. 885-888, 2013.

[14] M. E. Ibrahim, "Prevalence of Acinetobacter baumannii in Saudi Arabia: risk factors, antimicrobial resistance patterns and mechanisms of carbapenem resistance," Annals of Clinical Microbiology and Antimicrobials, vol. 18, no. 1, p. 1, 2019.

[15] Clinical and Laboratory Standards Institute (CLSI), Performance Standards for Antimicrobial Susceptibility Testing - 27th Edition. CLSI Document M100-27, CLSI, Wayne, PA, USA, 2017.

[16] R. N. Jones, T. R. Anderegg, J. M. Swenson, and Quality Control Working Group, "Quality control guidelines for testing gram-negative control strains with polymyxin B and colistin (polymyxin E) by standardized methods," Journal of Clinical Microbiology, vol. 43, no. 2, pp. 925-927, 2005.

[17] A. -P. Magiorakos, A. Srinivasan, R. B. Carey et al., "Multidrug-resistant, extensively drug-resistant and pandrug-resistant bacteria: an international expert proposal for interim standard definitions for acquired resistance," Clinical Microbiology and Infection, vol. 18, no. 3, pp. 268-281, 2012.

[18] N. Woodford, M. Ellington, J. Coelho et al., "Multiplex PCR for genes encoding prevalent OXA carbapenemases in Acinetobacter spp," International Journal of Antimicrobial Agents, vol. 27, no. 4, pp. 351-353, 2006.

[19] J. G. M. Koeleman, J. Stoof, M. W. van der Bijl, C. M. J. E. Vandenbroucke-Grauls, and P. H. M. Savelkoul, "Identification of epidemic strains of Acinetobacter baumannii by integrase gene PCR," Journal of Clinical Microbiology, vol. 39, no. 1, pp. 8-13, 2001.

[20] K. Vanbroekhoven, A. Ryngaert, P. Wattiau, R. Mot, and D. Springael, "Acinetobacter diversity in environmental samples assessed by $16 \mathrm{~S}$ rRNA gene PCRâDGGE fingerprinting," FEMS Microbiology Ecology, vol. 50, no. 1, pp. 37-50, 2004.

[21] A. Cherkaoui, S. Emonet, G. Renzi, and J. Schrenzel, "Characteristics of multidrug-resistant Acinetobacter baumannii strains isolated in Geneva during colonization or infection," Annals of Clinical Microbiology and Antimicrobials, vol. 14, p. 1, 2015.

[22] G. J. Silva, M. r. Correia, C. Vital et al., "Molecular characterization ofblaIMP-5, a new integron-borne metallo- $\hat{\mathrm{I}}^{2}$-lactamase gene from anAcinetobacter baumanniinosocomial isolate in Portugal," FEMS Microbiology Letters, vol. 215, no. 1, pp. 33-39, 2002.

[23] T. Naas, A. Ergani, A. Carrër, and P. Nordmann, "Real-time PCR for detection of NDM-1 carbapenemase genes from spiked stool samples," Antimicrobial Agents and Chemotherapy, vol. 55, no. 9, pp. 4038-4043, 2011.

[24] O. Cardoso, R. Leitão, A. Figueiredo, J. C. Sousa, A. Duarte, and L. V. Peixe, "Metallo- $\beta$-Lactamase VIM-2 in clinical isolates of Pseudomonas aeruginosafrom Portugal," Microbial Drug Resistance, vol. 8, no. 2, pp. 93-97, 2002.

[25] C. Héritier, L. Poirel, and P. Nordmann, "Cephalosporinase over-expression resulting from insertion of ISAba1 in Acinetobacter baumannii," Clinical Microbiology and Infection, vol. 12, no. 2, pp. 123-130, 2006.
[26] O. Oikonomou, S. Sarrou, C. C Papagiannitsis et al., "Rapid dissemination of colistin and carbapenem resistant Acinetobacter baumannii in central Greece: mechanisms of resistance, molecular identification and epidemiological data," BMC Infectious Diseases, vol. 15, p. 559, 2015.

[27] M. Lowings, M. M. Ehlers, A. W. Dreyer, and M. M. Kock, "High prevalence of oxacillinases in clinical multidrug-resistant Acinetobacter baumannii isolates from the Tshwane region, South Africa - an update," BMC Infectious Diseases, vol. 14, p. 521, 2015.

[28] M. M. Kock, A. N. Bellomo, N. Storm, and M. M. Ehlers, "Prevalence of carbapenem resistance genes inAcinetobacter baumanniiisolated from clinical specimens obtained from an academic hospital in South Africa," Southern African Journal of Epidemiology and Infection, vol. 28, no. 1, pp. 28-32, 2013.

[29] O. Perovic and V. Chetty, "Antimicrobial Resistance Surveillance from sentinel public hospitals, South Africa," Commun Dis Surveill Bull, vol. 14, pp. 56-67, 2015.

[30] W. Tunyapanit, P. Pruekprasert, K. Laoprasopwattana, and S. Chelae, "Antimicrobial susceptibility of Acinetobacter baumannii isolated from hospital patients," ScienceAsia, vol. 40, no. 1, pp. 28-34, 2014.

[31] R. Guckan, C. Kilinc, A. D. Demir, A. Capraz, and K. Yanik, "Antimicrobial susceptibility of Acinetobacter baumannii complex isolated from different clinical samples in A tertiary care hospital," Journal of Antibiotics Research, vol. 1, no. 1, p. 103, 2015.

[32] P. R. Joshi, M. Acharya, T. Kakshapati, U. Leungtongkam, R. Thummeepak, and S. Sitthisak, "Co-existence of blaOXA23 and blaNDM-1 genes of Acinetobacter baumannii isolated from Nepal: antimicrobial resistance and clinical significance," Antimicrobial Resistance \& Infection Control, vol. 6, p. $21,2017$.

[33] A. Howard, M. O’Donoghue, A. Feeney, and R. D. Sleator, "Acinetobacter baumannii," Virulence, vol. 3, no. 3, pp. 243-250, 2012.

[34] T. L. Luo, A. H. Rickard, U. Srinivasan, K. S. Kaye, and B. Foxman, "Association of blaOXA-23 and bap with the persistence of Acinetobacter baumannii within a major healthcare system," Frontiers in Microbiology, vol. 6, p. 182, 2015.

[35] B. A. Evans and S. G. B. Amyes, "OXA -Lactamases," Clinical Microbiology Reviews, vol. 27, no. 2, pp. 241-263, 2014.

[36] T. P. G. Chagas, K. R. Carvalho, I. C. de Oliveira Santos, A. P. D. A. Carvalho-Assef, and M. D. Asensi, "Characterization of carbapenem-resistant Acinetobacter baumannii in Brazil (2008-2011): countrywide spread of OXA-23-producing clones (CC15 and CC79)," Diagnostic Microbiology and Infectious Disease, vol. 79, no. 4, pp. 468-472, 2014.

[37] S. Ji, Y. Chen, Z. Ruan et al., "Prevalence of carbapenemhydrolyzing class D $\beta$-lactamase genes in Acinetobacter spp. isolates in China," European Journal of Clinical Microbiology \& Infectious Diseases, vol. 33, no. 6, pp. 989-997, 2014.

[38] G. Revathi, L. K. Siu, P.-L. Lu, and L.-Y. Huang, "First report of NDM-1-producing acinetobacter baumannii in east Africa," International Journal of Infectious Diseases, vol. 17, no. 12, pp. e1255-e1258, 2013.

[39] H. Salimizand, N. Noori, Z. Meshkat, K. Ghazvini, and S. J. Amel, "Prevalence of Acinetobacter baumannii harboring ISAba1/blaOXA-23-like family in a burn center," Burns, vol. 41, no. 5, pp. 1100-1106, 2015.

[40] I. Goic-Barisic, B. Bedenic, M. Tonkic et al., "Occurrence of OXA-107 and ISAba1 in carbapenem-resistant isolates of 
Acinetobacter baumannii from Croatia," Journal of Clinical Microbiology, vol. 47, no. 10, pp. 3348-3349, 2009.

[41] P. Martínez and S. Mattar, "Imipenem-resistant Acinetobacter baumannii carrying the ISAba1-bla OXA-23, 51 and ISAba1-bla ADC-7 genes in Monteria, Colombia," Brazilian Journal of Microbiology, vol. 43, no. 4, pp. 1274-1280, 2012.

[42] S. Vijayakumar, S. Anandan, D. Prabaa et al., "Insertion sequences and sequence types profile of clinical isolates of carbapenem-resistant A. baumannii collected across India over four year period," Journal of Infection and Public Health, no. 19 , pp. 30361-30362, 2019, In press.

[43] F. M. Elabd, M. S. Z. Al-Ayed, A. M. Asaad et al., "Molecular characterization of oxacillinases among carbapenem-resistant Acinetobacter baumannii nosocomial isolates in a Saudi hospital," Journal of Infection and Public Health, vol. 8, no. 3, pp. 242-247, 2015. 